\title{
Determinación de los niveles de colesterol LDL en ganado bovino comparando dos métodos
}

\author{
DETERMINATION OF LDL CHOLESTEROL LEVELS IN CATTLE COMPARING TWO METHODS
}

José Henry Osorio ${ }^{1,2,4}$, Jorge Abel Castañeda ${ }^{3}$

\section{Resumen}

El objetivo del estudio fue comparar el método Directo con el método de Precipitación para la determinación de colesterol LDL en una especie con patrón metabólico HDL. Se tomaron muestras de sangre de 56 vacas lecheras a primera hora del día. Se determinaron los niveles de colesterol LDL en el suero mediante ambos métodos utilizando kits comerciales. Los resultados fueron analizados estadísticamente mediante un análisis de varianza de una vía. El método directo reportó valores en $\mathrm{mg} / \mathrm{dl}$ de 46.66, 6.50, 101.83, 95.33 y 24.15 para promedio, mínimo, máximo, rango y desviación estándar, respectivamente y mediante el método de precipitación se obtuvieron valores (mg/dl) de 14.33, 0.36, $59.12,58.76$ y 11.21 , respectivamente, habiendo diferencia estadísticamente significativa entre estos métodos para un nivel de confianza del 95.0\%. Los resultados indican que el método directo es el más confiable para la determinación de los niveles de colesterol LDL.

Palabras clave: colesterol LDL; bovinos; método directo; método de precipitación

\section{Abstract}

The aim of this study was to compare the direct method with the precipitation method for the determination of LDL cholesterol in a species with a metabolic HDL pattern. Blood samples were taken from 56 dairy cows early in the day. The LDL cholesterol levels were determined in the serum by both methods using commercial kits. The results were statistically analyzed using a one-way analysis of variance. The direct method reported values (mg/dl) of 46.66, 6.50, 101.83, 95.33 and 24.15 for average, minimum, maximum,

\footnotetext{
${ }^{1}$ Laboratorio de Investigación en Bioquímica Clínica y Patología Molecular, Departamento de Ciencias Básicas de la Salud, Universidad de Caldas, Manizales, Colombia

${ }^{2}$ Laboratorio de Investigación en Metabolismo, Universidad de Manizales, Colombia

${ }^{3}$ Facultad de Ciencias Exactas y Naturales, Universidad de Caldas, Manizales, Colombia

${ }^{4}$ E-mail: jose.osorio_o@ucaldas.edu.co
}

Recibido: 12 de mayo de 2017

Aceptado para publicación: 5 de octubre de 2017 
range and standard deviation, respectively, and using the precipitation method values $(\mathrm{mg} / \mathrm{dl})$ of $14.33,0.36,59.12,58.76$ and 11.21 respectively, with a statistically significant difference between these methods for a $95.0 \%$ confidence level. The results indicate that the direct method is the most reliable for the determination of LDL cholesterol levels.

Key words: LDL cholesterol; cattle; direct method; precipitation method

\section{INTRODUCCIÓN}

Los lípidos en el plasma se encuentran en su totalidad asociados con proteínas formando complejos lipoproteicos llamados lipoproteínas (LP) que aseguran su transporte. Existen diferentes tipos de LP que difieren entre sí por su composición, tanto en sus componentes lipídicos como proteicos, clasificándose según su densidad en cinco grupos: quilomicrones (Q), LP de muy baja densidad (VLDL), LP de baja densidad (LDL), LP de densidad intermedia (IDL) y LP de alta densidad (HDL) (Attie, 2004).

El colesterol se halla principalmente formando la LDL junto a la apoproteína B-100, estando relacionado con el transporte de lípidos endógenos (Aranda et al., 2012). El hígado es el nodo central desde donde se redistribuye el flujo de lípidos hacia todo el organismo, además de ser allí donde se ensamblan las VLDL con el único objetivo de distribuir las fracciones lipídicas a los demás órganos (Kaneko, 1989), bajo la forma de LDL; es por ello que la capacidad de movilización de estas fracciones es de vital importancia, tanto para redistribuir las reservas de energía como para impedir la acumulación de grasa en el hígado (Galvis et al., 2003), y de esta manera, no ser afectado con el aumento de grasas y colesterol en la dieta, especialmente de colesterol HDL (Bauer, 1997). Los bovinos tienen un metabolismo inverso a los humanos, pues estos últimos presentan mayores niveles de colesterol LDL como respuesta a raciones altas de grasa (Coppo et al., 2003).
Las hormonas tiroideas tienen importancia en la regulación de los niveles de colesterol, ya que afectan el metabolismo de los lípidos disminuyendo los niveles de colesterol a través de un aumento en la absorción celular de las LDL y de un incremento en la degradación de colesterol y las LDL (Osorio, 2016). Estos efectos se presentan en estados fisiopatológicos relacionados con hipersecreción de hormonas tiroideas o en estado de deficiencia tiroidea donde se puede observar hipercolesterolemia (Osorio, 2017). Por otro lado, la elevación de los estrógenos en vacas lactantes al final de la gestación promueve un aumento en los receptores de LDL, disminuyendo las concentraciones plasmáticas de estas LP (Rayssiguier et al., 1988). Los niveles de estrógenos disminuyen después del parto, lo que conlleva a una baja en los receptores de LDL y, por consiguiente, un aumento paulatino en la concentración plasmática de LDL durante la lactancia temprana (Osorio y Vinazco, 2010).

El incremento simultáneo en las concentraciones plasmáticas de colesterol total, LDL y HDL, se debe al aumento de los niveles de colesterol durante la lactancia y este hecho hace que también se eleven los niveles de apolipoproteínas, las cuales forman parte tanto de las HDL como de las LDL, representando cerca del $50 \%$ del incremento en lípidos séricos dados en dicho periodo (Raphael et al., 1973). Por estas razones, al momento de realizar las mediciones de las LP se debe tener en cuenta tanto el estado productivo como el catabolismo de las VLDL. 
El objetivo del presente estudio fue comparar el método directo con el método de precipitación para la determinación de los niveles de colesterol LDL en bovinos, teniendo en cuenta que su patrón metabólico es el HDL.

\section{Materiales y Métodos}

El experimento se realizó en el departamento de Caldas, Colombia. El clima se caracteriza por ser templado frío, con precipitación anual entre 1500 y $3000 \mathrm{~mm}$, temperatura media de $21{ }^{\circ} \mathrm{C}$ y humedad relativa de $66 \%$.

Se seleccionaron 56 hembras Bos taurus (Holstein, Normando y Blanco Orejinegro [BON]) sin discriminación de edad. Los animales eran criados bajo un sistema semi-intensivo, presentaban un adecuado plan sanitario (desparasitados y con vacunas al día), y tenían condición corporal entre 3 y 4 (escala $1-5)$.

La toma de muestras de sangre se hizo mediante venopunción yugular entre las 06:00 y las 08:00 h. Los animales habían quedado en ayunas para la toma de las muestras. Las muestras fueros refrigeradas para su transporte al laboratorio, donde fueron centrifugadas (centrifuga Thermo IEC CL31 Multispeed) a $2579 g$ durante 15 minutos. E1 suero fue extraído para determinar los niveles de colesterol LDL utilizando kits comerciales (BioSystems $\left.{ }^{\circledR}\right)$ mediante el método directo y el método de precipitación. La lectura de los resultados se realizó en un analizador semiautomático de química serial Rayco.

En el método directo (Wu, 2006), un detergente específico solubiliza el colesterol de las HDL, VLDL y los quilomicrones. Los ésteres de colesterol son hidrolizados por el colesterol esterasa y el colesterol oxidasa mediante una reacción no formadora de color. Un segundo detergente solubiliza el colesterol de las LDL de la muestra, el cual se cuantifica espectrofotométricamente.

En el método de precipitación, las LDL presentes en la muestra precipitan en presencia de polivinil sulfato. La concentración de colesterol LDL se calcula por diferencia entre los valores de colesterol en el suero y en el sobrenadante obtenido tras la precipitación, donde el colesterol se cuantifica espectrofotométricamente.

Se obtuvo el cálculo del promedio, rango, mínimo, máximo, varianza y desviación estándar para la cuantificación del colesterol LDL, tanto por el método directo como por el método precipitado. Se evaluaron las diferencias sobre los dos métodos por medio de un análisis de varianza utilizando el programa Stat Graphics Plus 5.1, aceptando diferencias estadísticamente significativas cuando $\mathrm{p}<0.05$.

\section{Resultados y Discusión}

Los valores de colesterol LDL mediante el método directo y el método precipitado se presentan en el Cuadro 1, habiendo diferencia significativa entre ambos métodos.

Las concentraciones plasmáticas de colesterol LDL tienen importancia en las hembras de producción lechera, ya que estos niveles aumentan a medida que avanza la lactancia. Bauchart (1993) afirma que los receptores para LDL se incrementan conforme aumentan los niveles de estrógenos. Es probable que las altas concentraciones de LDL durante el posparto sea un fenómeno necesario para su transporte y distribución (Osorio et al., 2010). Henao-Restrepo et al. (2010) reportaron que el aumento de las concentraciones plasmáticas de LDL tiene un efecto indirecto en la disminución de sus receptores encontrando una asociación inversa con la condición corporal, y que está ultima se redujo a medida que avanzó la lactancia. Es por ello que deben realizarse eva- 
Cuadro 1. Valores de colesterol LDL (mg/dl) en bovinos Bos taurus

\begin{tabular}{lccc}
\hline Parámetros & Método precipitado & Método directo & P-valor \\
\hline Promedio & 14.33 & 46.66 & \\
Desviación estándar & 11.21 & 24.15 & 0.0000 \\
Mínimo & 0.36 & 6.50 & \\
Máximo & 59.12 & 101.83 & \\
\hline
\end{tabular}

luaciones de las LP para obtener un mejor conocimiento sobre el estado fisiológico y productivo de los bovinos, siguiendo la recomendación del National Cholesterol Education Program (NCEP). Asimismo, la relación entre el colesterol LDL y el colesterol HDL demuestra ser tres veces más sensible que el obtenido al determinar únicamente colesterol total (Demacker et al., 1984).

Existen muchas controversias en los métodos usados para la medición de las LP. Los métodos de ultracentrifugación requieren una técnica de separación que, aparte de ser tedioso, consume mucho tiempo; además, las concentraciones de LP pueden ser sustancialmente alteradas por las fuerzas centrífugas y la contaminación cruzada puede ser común. Asimismo, el uso de diferentes tipos de equipos y materiales, así como de la experiencia de los usuarios, pueden afectar los resultados (Guidance for Industry, 2001). Sin embargo, mucho se corrige con los métodos homogéneos automatizados que utilizan reactivos específicos para ser más selectivos y miden directamente el colesterol asociado a LDL (Nauck et al., 2002). No obstante, los métodos directos y de precipitación son los más practicados en los laboratorios (BioSystems, 2016, 2017).

Un estudio realizado en humanos comparó el método directo con la fórmula de Friedewald para la medición de LDL (Mendes
De Cordoba et al., 2004), no encontrando diferencias significativas; sin embargo, los autores indicaron que el método directo es el más apropiado para el laboratorio de rutina, ya que no tiene las interferencias que presentan los métodos con precipitación. De igual forma, Osorio y Uribe-Velásquez (2011) evaluaron los mismos métodos en la especie equina, confirmando lo reportado en el humano, y señalando, igualmente, que el método directo es el más confiable, aunque presenta el inconveniente de los altos costos que representa su ejecución. Por otro lado, este estudio reporta resultados contradictorios con el estudio de Osorio et al. (2010) quienes reportaron una correlación positiva entre los métodos directo y precipitado.

Con base a los resultados obtenidos, no se recomienda el método de precipitación para la medición de colesterol LDL en bovinos. El método directo es más costoso pero es más confiable para este propósito.

\section{Literatura Citada}

1. Aranda MV, Brave N, Casagrande R. 2012. Colesterol en bovinos. Sitio Argentino de Producción Animal. [Internet]. Disponible en: http:// www.produccionanimal.com.ar/informacion_tecnica/carne_y_subproductos/26colesterol_en_bovinos.pdf 
2. Attie AD. 2004. Lipoprotein/cholesterol metabolism. In: Meyers RA (ed). Encyclopedia of physical science and technology. $3^{\text {rd }}$ ed. Madison, Wisconsin, USA: Academic Press. p. 643-660.

3. Bauchart D. 1993. Lipid absorption and transport in ruminants. J Dairy Sci 76: 3864-3881. doi: 10.3168/jds.S00220302(93)77728-0

4. Bauer JE. 1997. Metabolismo comparado de lípidos y lipoproteínas. Pet's Sci 13:362-376.

5. BioSystems. 2016. Colesterol LDL Directo Detergente. [Internet]. Disponible en: http://www.biosystems.es/productsfilt.aspx?keyw=ldl

6. BioSystems. 2017. Colesterol LDL Reactivo Precipitante. [Internet]. Disponible en: http://www.biosystems.es/ productsfilt.aspx?keyw=ldl

7. Coppo NB, Coppo JA, Lazarte MA. 2003. Intervalos de confianza para colesterol ligado a lipoproteínas de alta y baja densidad en suero de bovinos, equinos, porcinos y caninos. Rev Vet 14 : 3-10.

8. Demacker PN, Higmans AG, Breminkmeijer BJ, Jansen AP, Van't Laar A. 1984. Five methods for determining low-density lipoprotein cholesterol compared. Clin Chem 30: 1797-1800.

9. Galvis RD, Correa HJ, Ramirez NF, Soler $W$. 2003. Influencia de las alteraciones hepáticas sobre la actividad PEPCK, la generación de IGF-I plasmático y la reactivación ovárica en vacas en la lactancia temprana. Rev Col Cienc Pec 16: 228-236.

10. Guidance for industry: bioanalytical method validation. 2001. US Department of Health and Human Services. [Internet]. Available in: https:/ /www.fda.gov/downloads/Drugs/ Guidance/ucm070107.pdf

11. Henao-Restrepo G, Galvis-Goez RD, Cardona-Bermúdez LM, CastroHenao NM. 2010. Relación entre pérdida de peso, perfil lipídico y concentraciones plasmáticas de leptina en vacas cebú primerizas. Rev Fac Nal Agr Medellín 63: 5595-5605.

12. Kaneko JJ. 1989. Clinical biochemistry of domestic animals. USA: University of California. $930 \mathrm{p}$.

13. Mendes de Cordova CM, Schneider CR, Juttel LD, Mendes de Cordova M. 2004. Avaliacáo da dosagem directa do colesterol-LDL em amostras de sangue de 10.664 pacientes em comparacáo com o uso da fórmula de Friedewald. Arq Bras Cardiol 83: 482-487.

14. Nauck M, Warnick R, Rifai N. 2002. Methods for measurement of LDLcholesterol: a critical assessment of direct measurement by homogeneous assays versus calculation. Clin Chem 48: 236-254.

15. Osorio J, Uribe-Velásquez LF. 2011. Comparación de los métodos directo y de Friedewald para la determinación de los niveles de colesterol LDL en el equino. Rev MVZ Córdoba 16: 2549-2553.

16. Osorio JH, Correa Carvajal D, Pérez JE. 2017. Concentraciones de hormona estimulante de la tiroides y tiroxina libre en ovinos jóvenes. Rev Med Vet. 33: 77-81. doi: 10.19052/mv.4055

17. Osorio JH, Rangel VR, Carmona, J. Barrera L, Vinazco J, Suarez, Y, Cañas $E$, Giraldo $S$, et al. 2010. Comparación de dos métodos para la determinación de los niveles de colesterol HDL, en una especie con patrón HDL. Vet Zootec 4: 19-22.

18. Osorio JH, Vinasco J. 2016. Comparación de los niveles séricos de TSH y T4 libre en bovinos. Rev Med Vet Zoot 63: 87-94. doi: 10.15446/rfmvz.v63$\mathrm{n} 2.59356$

19. Osorio JH, Vinazco J. 2010. El metabolismo lipídico bovino y su relación con la dieta, condición corporal, estado productivo y patologías asociadas. Biosalud 9: 56-66.

20. Raphael B, Dimick P, Puppione D. 1973. Lipid characterization of bovine serum lipoproteins throughout gestation and lactation. J Dairy Sci 56: 1025-1032. doi: $10.3168 /$ jds.S0022-0302(73)85300-7 
21. Rayssiguier Y, Mazur A, Gueux E. 1988. Plasma lipoprotein and fatty liver in dairy cows. Res Vet Sci 45: 389-393.
22. Wu A. 2006. Tiez Clinical guide to laboratory tests. $4^{\text {th }}$ ed. New York: Saunders Elsevier. 1798 p. 